$\mathbf{m} / \mathbf{S}$

médecine/sciences $1997 ; 13: 143-4$

\title{
MÉDECINE ET SCIENCE AUX ÉTATS-UNIS
}

\section{Axel Kahn}

\section{ADRESSE}

A. Kahn : directeur de l'U. 129 de l'Inserm, directeur du Laboratoire de Génétique et Pathologie Moléculaires. Inserm U. 129, Institut Cochin de Génétique Moléculaire, 24, rue du Faubourg-Saint-Jacques, 75014 Paris, France.

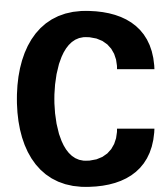
e numéro de médecine/ sciences est exceptionnel à plus d'un titre. Tout d'abord, par son objet: son unité première est de présenter différentes facettes du meilleur de la création scientifique de francophones installés aux ÉtatsUnis d'Amérique. Le choix des contributions aurait, naturellement, pu être tout à fait différent et, dans l'avenir, il pourra l'être car nous avons conscience de n'avoir qu'effleuré le sujet des réalisations scientifiques et médicales remarquables des francophones installés aux États-Unis. Dans ce numéro, c'est manifestement la biologie du développement qui est à l'honneur, ce qui n'étonnera pas les lecteurs de médecine/sciences qui savent que celleci est à l'origine de beaucoup des progrès essentiels réalisés ces dernières années en biologie générale, y compris dans ses applications médicales; il suffit de penser à ce qu'a apporté cette discipline à la compréhension des phénomènes de l'apoptose, du cancer et de nombreuses maladies pour s'en convaincre. Ce choix a également une autre raison, plus circonstancielle: ce numéro a initialement été imaginé en concertation étroite avec Claude Desplan, luimême éminent biologiste du développement.

Au-delà de cette science superbe qui nous est montrée, Roger Guillemin et Claude Desplan s'interrogent sur les conditions qui l'ont rendue possible et ont même très puissamment contribué à son développement. Roger Guillemin rappelle que, dès la période d'avant-guerre et, plus encore, dans celle qui a suivi le second conflit mondial, l'Amérique s'est révélée une terre d'accueil extrêmement généreuse pour tous les scientifiques du monde, ne limitant d'ailleurs pas cette générosité au territoire national puisque des fonds américains ont financé des équipes scientifiques d'excellence un peu partout dans le monde, et notamment en Europe. C'est que la culture américaine a très tôt intégré la dimension scientifique et technologique, même si cela s'est parfois fait dans une atmosphère rien moins que consensuelle : que l'on pense, par exemple, aux épiques débats entre l'évolutionnisme darwinien et le créationnisme, qui sont encore loin d'être totalement apaisés.

La conviction des Etats-Unis que leur avenir et leur puissance dépendent de la science et des techniques que le pays engendre explique ce paradoxe : dans ce pays de la libre entreprise et de l'initiative privée, le soutien à la recherche par les autorités fédérales ne s'est pratiquement jamais démenti et, après les craintes nées il y a environ deux ans du changement de majorité politique au Congrès, ne semble pas se démentir 
en ce début du deuxième mandat du Président Clinton. D'autres facteurs du succès résident, en revanche, dans l'application très large du principe de la libre compétition scientifique, des moyens étant assurés aux meilleurs, quels que soient leur âge et leurs appuis. Dans le domaine de la recherche scientifique, où la dimension de la compétition va de soi, ce principe ne peut que donner de bons résultats, d'autant plus que les ÉtatsUnis disposent d'une masse considérable de jeunes scientifiques, ceux d'Amérique mais aussi les stagiaires postdoctoraux en provenance du monde entier. Comme en matière sportive, l'élargissement de la base et la sévérité d'une sélection honnête, ne peuvent qu'aboutir à d'excellents résultats, renforcés ici par la position économique des États-Unis. Claude Desplan insiste sur les tracas qui attendent les jeunes français formés à cette école exigeante lorsqu'ils veulent revenir dans leur pays : bien souvent, ils doivent observer alors que leur qualité ne suffit plus, et que leur seront opposés leur âge, la faiblesse des soutiens dont ils disposent dans un système encore très marqué par le mandarinat ou, lorsqu'ils viennent de certains très grands laboratoires, la longue liste d'attente des étudiants formés dans ce site et qui attendent de décrocher une position. C'est d'ailleurs cette situation qui explique en partie le nombre d'excellents scientifiques francophones faisant toute leur carrière, ou au moins la plus grande partie de celle-ci, aux Etats-Unis. Un troisième aspect par lequel ce numéro est remarquable est le rapprochement qu'il autorise entre un succès incontestable et incontesté - la science américaine- et un succès ou un échec, comme on le voudra, relatifs - la santé. Bettina Experton, médecin français vivant aux ÉtatsUnis depuis 15 ans et spécialiste de l'évolution des systèmes de santé dans ce pays, nous trace un tableau précis et sans complaisance de la situation actuelle.

Les principes en sont connus : le marché de la santé appartient au monde de la libre entreprise et, ici, l'aide publique se limite à des catégories particulières : personnes âgées et risées. Ces principes ont abouti à faire de la médecine américaine l'une des plus innovante du monde sur les plans technologique et thérapeutique... mais aussi la plus chère, alors même que ses résultats globaux en terme de soins pour tous, voire même de longévité générale et de mortalité infantile, restent inférieurs à ce qu'ils sont au Canada, au Japon ou dans la plupart des pays européens. L'exigence d'une maîtrise des dépenses de santé a conduit au développement d'un système qui a progressivement tourné le dos à tous les principes de la médecine libérale tels qu'ils sont encore défendus avec vigueur dans notre pays. Pour plus de la moitié des américains, la santé est dispensée par l'intermédiaire d'organisations capitalistes très rentables avec lesquelles les employeurs passent un accord. Les médecins travaillent eux-mêmes sous contrat avec ces organisations (les $\mathrm{HMO}^{*}$ ) et leur activité est très étroitement réglementée. L'accès aux spécialistes n'est possible qu'après orientation par un généraliste, et d'importantes limitations sont mises à l'utilisation par ces derniers des techniques et des traitements les plus coûteux. Le résultat de cette évolution est singulier: globalement, les dépenses de santé n'ont guère diminué, mais, en revanche, les organisations précitées se sont considérablement enrichies, et acquièrent progressivement un quasi-monopole dans le financement et la distribution des soins, en venant même à racheter certains des grands centres hospitaliers universitaires. Les salariés américains, dont la majorité est peu consommatrice de soins, ne peuvent qu'être satisfaits $d u$ système, mais il fait peser des menaces sur la qualité du traitement des cas les plus coûteux et, accessoirement, transforme profondément les conditions d'exercice médical des praticiens. De plus, 41 millions d'Américains ne sont pas - et parfois ne sont plus - couverts par un système d'assurance maladie. Une leçon que l'on peut tirer de ce survol de la science et de la médecine

$\overline{* H M O: \text { Health }}$ Maintenance Organizations. américaine, dont ce numéro de médecine/sciences nous donne l'occasion, est que les principes qui président aux succès de l'une peuvent ne pas tous valoir intégralement pour l'organisation optimale de l'autre.

Ainsi, l'exigence de solidarité n'a évidemment pas de sens, au niveau national, dans un système où la sélection du meilleur scientifique s'impose clairement. En revanche, ce principe ne peut être évacué dès lors que l'on traite de ce que l'Organisation Mondiale de la Santé considère comme l'un des droits les plus fondamentaux de l'Homme : «La possession du meilleur état de santé qu'il est capable d'atteindre $(. . . / .$.$) , quelles que soient$ sa race, sa religion, ses opinions publiques, sa condition économique et sociale » [1]. Même si ce principe comporte sa part d'utopie, notamment du fait de la définition large de la santé par l'OMS ( « un état de complet bien-être physique, mental et social (... et non pas seulement...) une absence de maladie ou d'infirmité ») [1], il relève bien qu'une des obligations essentielles des sociétés humaines, en fait l'une de leurs justifications, est de s'efforcer d'assurer aux personnes un accès égal aux soins les mieux adaptés aux différentes situations, en fonction du progrès des connaissances et des techniques. Le défi est dès lors clairement lancé : la meilleure science possible, pour une meilleure médecine... pour tous

\section{RÉFÉRENCE}

1. Préambule à la constitution de l'OMS, 22 juillet 1946.

\section{TIRÉS À PART}

A. Kahn. 